\title{
Argentina: Dinámica reciente del sector de software y servicios informáticos
}

\author{
Florencia Barletta, Mariano Pereira, Verónica Robert \\ y Gabriel Yoguel
}

RESUMEN

Bajo un marco teórico evolucionista, se estudia la repercusión de las capacidades organizacionales y de absorción y de las vinculaciones en el desempeño de firmas argentinas de software y servicios informáticos. Los modelos estimados denotan que los resultados de innovación dependen de la gestión de calidad, de la existencia de equipos de investigación y desarrollo (I+D) y de posiciones intermedias en la red de vinculaciones. El desempeño económico no puede abordarse sobre la base de un mismo conjunto de indicadores. Mientras que la productividad se explica por la calificación de los recursos humanos y el crecimiento del empleo por la presencia de equipos de I+D, la probabilidad de exportar se relaciona con disponer de certificaciones, adoptar metodologías ágiles y ocupar posiciones intermedias en la red. La heterogeneidad en los modelos de negocios sugiere que no es posible establecer una relación única entre innovación y desempeño para el conjunto de firmas.

PALABRAs Claves

CLASIFICACIÓN JEL

AUTORES
Industria, informática, programas computacionales, comercio de servicios, innovaciones, investigación y desarrollo, gestión de calidad, medición, evaluación, Argentina

O14, O30, L86

Florencia Barletta es investigadora-docente del Instituto de Industria de la Universidad Nacional de General Sarmiento.mfbarletta@gmail.com

Mariano Pereira es investigador-docente del Instituto de Industria de la Universidad Nacional de General Sarmiento.yomarianopereira@yahoo.com.ar

Verónica Robert es investigadora-asistente del Consejo Nacional de Investigaciones Científicas y Técnicas (CONICET) e investigadora-docente del Instituto de Industria de la Universidad Nacional de General Sarmiento. vrobert@gmail.com

Gabriel Yoguel es investigador-docente del Instituto de Industria de la Universidad Nacional de General Sarmiento y coordinador del Programa de Investigación de Economía del Conocimiento (PIEc). gyoguel@gmail.com

Todos los autores forman parte del Programa de Investigación de Economía del Conocimiento (PIEC) www.ungs.edu.ar/piec 


\section{I}

\section{Introducción}

Desde la devaluación del año 2002, el sector de software y servicios informáticos (sSI) ha sido uno de los sectores de mayor crecimiento en la economía argentina. Su dinámica agregada entre 2003 y 2010 muestra incrementos en el empleo, las ventas y las exportaciones - un promedio anual de $17,8 \%, 17,6 \%$ y $21,4 \%$, respectivamente (OEDE, 2012; CESSI, 2012) - muy superiores a los de la industria manufacturera en su conjunto $(3,8 \%, 8,3 \%$ y $13,0 \%$, respectivamente $)^{1}$, destacándose también en la región ${ }^{2}$. Este desempeño se explicaría por la mayor

$\square$ Este trabajo constituye un ulterior desarrollo del informe final del proyecto "Capacidades de absorción y conectividad en sistemas productivos y de innovación locales", financiado por la Fundación Carolina de España.

1 A pesar del fuerte crecimiento del sector de ssI en la Argentina desde la devaluación de 2002, es importante señalar los esfuerzos realizados por el Estado en las décadas previas, que acompañaron los avances y retrocesos de la economía argentina desde los años sesenta (Aspiazu, Basualdo y Nochteff, 1986 y 1990; Babini, 2003). Hacia 1962, un equipo de investigación en ingeniería construyó la primera computadora argentina denominada Census, lo que dio lugar a la construcción de otros dos prototipos en esa década. Hacia mediados de la década de 1960 la Argentina había hecho importantes avances en informática en el contexto latinoamericano. En esa etapa infantil del sector, tanto en la Argentina como en el mundo, el desarrollo del software estaba directamente ligado al hardware. En los primeros años del decenio de 1970, la IBM instala una planta de producción de impresoras y otros periféricos. La crisis de la industrialización mediante sustitución de importaciones (ISI) y el proceso de apertura del período 1976-1983 tuvieron consecuencias negativas para el desarrollo del sector. Luego de la crisis de la deuda (1982), entre 1983 y 1991 la política se focalizó en el diseño de subsidios orientados al sector y a la creación de un programa de investigación argentino-brasileño en informática que pusieron de relieve la necesidad de formar recursos humanos de elevada calificación en el área de software. Como consecuencia, en 1986 se creó la Escuela Superior Latinoamericana de Informática (ESLAI), disuelta en 1991. Durante la fase de apertura y desregulación de los mercados (1991-2001) predominó la idea de que el progreso técnico incorporado, consistente en la importación de hardware y software, produciría derrames de conocimientos en toda la estructura productiva. Con anterioridad a la devaluación de 2002, las ventas internas y externas, el empleo y la productividad del sector de sSI argentino eran muy poco relevantes si se los comparaba con los de los países de mayor desarrollo relativo y con los casos de la India, Irlanda e Israel (Arora y Gambardella, 2005; López y Ramos, 2007; OCDE, 2002).

${ }^{2}$ La ocupación de ssi en la Argentina duplicaba en 2003 la registrada en el Uruguay y la quintuplicaba en 2010 (CUTI, 2012; CESSI, 2012). En relación con el Brasil, la ocupación de ssi en la Argentina, que alcanzaba al $14 \%$ de la brasileña en 2003, pasa a ser del $21,5 \%$ en 2010 (SOFTeX, 2012; RAIS, 2012; Oede/MTss, 2012). Por su parte, el peso de las exportaciones en las ventas en 2010 (19\%) se ubica en un lugar intermedio entre el Brasil (5\%) y el Uruguay (39\%), y es significativamente menor que el de la India (76\%), Irlanda (85\%) e Israel (73\%). competitividad de la economía argentina a partir de la devaluación y el crecimiento del mercado interno, junto con la generalización de la subcontratación de servicios externos (outsourcing) y el desarrollo de software a nivel global, que permitió dinamizar un segmento importante de las firmas de ssI. Asimismo, desde 2004 está vigente un conjunto de políticas públicas que promueven la certificación de calidad, la actividad exportadora y los esfuerzos de innovación. Dicho conjunto comprende la creación del Fondo Fiduciario de Promoción de la Industria del Software (FONSOFT) en el marco de la Ley de Promoción de la Industria del Software, promulgada en 2004, el desarrollo de infraestructura y la promoción de las carreras de informática en la Argentina (Zuckerfeld y otros, 2012). Tales políticas públicas se impulsaron bajo la premisa de que las mejoras en las capacidades organizacionales de las firmas y los esfuerzos de innovación conducen a un más elevado desempeño innovador, que repercute en los niveles productivos de las firmas en materia de crecimiento del empleo, la productividad y las exportaciones.

Sin embargo, a diferencia de la dinámica de la industria manufacturera durante el período, el fuerte crecimiento del empleo, de las exportaciones, e incluso de la razón entre las exportaciones y las ventas no se vio reflejado en mejores niveles relativos de productividad en las firmas de sSI. A su vez, los resultados - en concordancia con los argumentos evolucionistas sobre capacidades e innovación- parecen obedecer a la complementación de fuentes internas y externas de conocimientos (desarrollo de capacidades organizacionales, capacidades de absorción y posición de las firmas en el espacio de conexiones), en un contexto de importantes diferencias dentro del sector en función de distintos modelos de negocios que favorecen de forma desigual a las conductas innovadoras (Yoguel y otros, 2004). Es decir, detrás del comportamiento agregado de expansión en las ventas, el empleo y las exportaciones, se esconden marcadas heterogeneidades que ponen de relieve que la relación entre capacidades, vinculaciones, innovación y desempeño productivo no parece verificarse en todos los casos y que la vinculación entre las diferentes medidas de desempeño es débil.

Por otra parte, la relevancia que adquieren los desarrollos a medida, la subcontratación externa y las estrategias de diversificación de actividades conduce a 
plantear - en servicios en general y en ssi en particularel alcance de la definición convencional de resultado de innovación que se deriva de las encuestas tecnológicas y que se conceptualiza como introducción de productos y procesos nuevos o mejorados. Esto lleva a discutir la importancia que adquiere el desarrollo de capacidades de las firmas no solo como determinante de los resultados de innovación, sino incluso como definición misma de la innovación en servicios.

En este marco se plantean las siguientes preguntas de investigación: ¿el contexto global, local y de políticas afectó de igual manera a todas las firmas del sector o, por el contrario, dentro del desempeño agregado se ocultan marcadas heterogeneidades vinculadas a distintos modelos de negocios, dispares capacidades y desigual conectividad? ¿En qué medida esta heterogeneidad se manifestó en impactos diferenciales en términos de productividad, empleo y exportaciones? Finalmente, ¿hasta qué punto el desarrollo de capacidades, la ubicación de las firmas en la red y las distintas estrategias comerciales de las firmas aparecen relacionados con los resultados de innovación?

En este contexto, el objetivo del presente artículo es estudiar las características del desarrollo reciente del sector de ssi, a partir de un marco teórico evolucionista en que se enfatiza el papel de las capacidades de absorción y organizacionales y el desarrollo de vinculaciones sobre la base del desempeño económico y de innovación de las firmas. Para ello se utilizó una base de 189 firmas del sector de ssi en la Argentina, que releva información para el período 2008-2010. En tal sentido, este artículo constituye un aporte clave para el estudio del desarrollo reciente del sector de ssi, ya que la mayor parte de los trabajos previos se centran o en estudios de caso o no incluyen la dinámica posdevaluación (Chudnovsky, López y Melitsko, 2001).

En la sección II se desarrolla el esquema conceptual del artículo, donde se combinan la literatura evolucionista de la innovación, el enfoque de redes y la literatura sobre innovación en servicios (Gallouj y Weinstein, 1997; Djellal y Gallouj, 2001; Coombs y Miles, 2000; Gallouj y Savona, 2009), de los que se derivan las hipótesis de trabajo. En la sección III se presenta una breve revisión de la literatura empírica sobre la innovación en el sector de SSI. En la sección IV se desarrolla la metodología utilizada, la construcción de los indicadores y la estadística descriptiva. En la sección V se discuten los principales resultados de los modelos estimados que permiten evaluar en qué medida las capacidades organizacionales y de absorción, la posición de las firmas en la arquitectura de la red y su heterogeneidad en términos de modelos de negocios permiten explicar los resultados de innovación y su desempeño. Finalmente, en la sección VI se plantean las principales conclusiones.

\section{II}

\section{Marco teórico e hipótesis}

En el marco teórico se combinan el enfoque evolucionista acerca de la naturaleza sistémica de la innovación (Nelson y Winter, 1982; Saviotti y Metcalfe, 1984; Silverberg, Dosi y Orsenigo, 1988; Dosi, 2000) con el enfoque de redes sociales aplicado a la circulación y generación de conocimiento (Powell, Koput y Smith-Doerr, 1996; Gulati, 1999; Barabási y Albert, 1999; Cowan y Jonard, 2004) y con la literatura en que se discuten las características de los procesos de innovación en servicios (Gallouj y Weinstein, 1997; Djellal y Gallouj, 2001; Coombs y Miles, 2000; Drejer, 2004; Gallouj y Savona, 2009).

En primer lugar, en el enfoque evolucionista los procesos de innovación se caracterizan como un fenómeno sistémico que no ocurre exclusivamente dentro de las firmas, sino que depende en gran medida de sus interacciones con otros agentes del sistema, como otras firmas e instituciones de fomento a la innovación: consultores, centros tecnológicos, cámaras empresariales y universidades. Tanto las capacidades de las firmas como las vinculaciones que estas establecen con el objetivo de incrementar sus capacidades inciden en las posibilidades de obtener innovaciones y de mejorar el desempeño productivo y económico. La innovación es entendida a partir de la complementación de saberes internos y externos a las firmas. El acceso a los conocimientos externos depende de los vínculos que las organizaciones establecen entre sí y del desarrollo de capacidades internas. Estas capacidades tienen que ver con los conceptos de capacidad de absorción, definida como la posibilidad de identificar, acceder a y aplicar conocimiento externo (Cohen y Levinthal, 1990), y capacidad organizacional, que se refiere a las habilidades para la codificación del 
conocimiento tácito a partir de los procesos de gestión de calidad y de las formas de organización del trabajo que operan con respecto a la circulación del conocimiento en el interior de la organización.

En este contexto, la innovación es el resultado de una dinámica no lineal en el proceso de aprendizaje, conducida por el reforzamiento mutuo entre las capacidades y la conectividad. Los contextos locales en que las firmas actúan juegan un papel clave para comprender sus conductas innovadoras. Estos incluyen el marco institucional, el contexto de políticas, los procesos de competencia, la posición de las firmas dentro de la red de conexiones determinada por sus vinculaciones directas e indirectas, y las características estructurales de esta red. En la medida en que la dinámica innovadora es el resultado de un proceso sistémico, el enfoque evolucionista de la innovación se complementa con el de redes.

El enfoque de redes aplicado a la teoría evolucionista de la innovación permitió poner de manifiesto la importancia de las vinculaciones indirectas y no solo directas con relación a los procesos de innovación. Existe una larga tradición dentro de la literatura evolucionista de la innovación en que se ha enfatizado la relevancia de las vinculaciones con distintos agentes e instituciones en el proceso innovativo (Freeman, 1991). En este marco, la literatura se centró mayormente en el análisis del impacto de los vínculos directos entre distintos agentes. Sin embargo, el estudio de las vinculaciones indirectas, es decir, de la conectividad de las firmas a partir de las vinculaciones de sus vecinos y de las características estructurales de la red global, es relativamente novedoso. Las investigaciones en que se incorporan estas dimensiones analíticas - vinculaciones indirectas y características de la red - para dar cuenta de los procesos de circulación y creación de conocimiento en redes organizacionales, se enmarcan en el enfoque de redes sociales (social networks) (Boschma y ter Wal, 2006; Giuliani y Bell, 2005).

En la literatura sobre redes inter-organizacionales y de innovación se ha debatido largamente la tensión entre difusión y creación de conocimiento (Cowan y Jonard, 2003; Watts, 2006). La alta densidad local de una red es positiva para la difusión de conocimiento, pero el proceso de introducción de novedad se ve afectado negativamente, ya que todos los agentes de este sector disponen de información completa. En contraparte, una red completamente aleatoria alimenta de novedad en forma constante a un entorno local específico, pero el proceso de difusión es más dificultoso. Un punto intermedio es aquel donde están presentes las características de las redes regulares de alta densidad local y las redes aleatorias donde los caminos son relativamente más cortos entre dos extremos cualesquiera. Estas son las redes de mundos pequeños, que refieren a aquellas redes donde "cliques" 3 de firmas se vinculan entre sí por medio de nodos que actúan como "puentes" (Watts, 2006). En este tipo de redes las posiciones intermedias se vinculan a la introducción de innovaciones y al mejor desempeño de las firmas en relación con las posiciones centrales y periféricas. Diversos autores (Watts 1999; Hargadon, 2003; Cowan y Jonard 2003; Cowan y Jonard 2004; Verspagen y Duysters, 2004; Schilling y Phelps, 2004) afirman que las redes de mundos pequeños estimulan el desarrollo de capacidades de las firmas y por ende mejoran su conducta innovadora. Las redes de mundos pequeños permiten la coexistencia de vinculaciones muy densas y agrupadas con otras vinculaciones más débiles cuyos nodos se encuentran más distantes en la red. Las vinculaciones más densas ayudan a promover la confianza y colaboración entre firmas, mientras que las relaciones con nodos distantes incorporan conocimiento nuevo y no redundante al núcleo (core) de la red. De esta manera, las redes de mundos pequeños contribuyen a una mejor movilización de información y a innovaciones, rutinas, experiencias y otros recursos que no circulan en el entorno local de la firma, mejorando su proceso de aprendizaje.

No obstante, el marco teórico discutido hasta aquí no se hace eco de las especificidades que pudieran existir en materia de innovación en servicios en general y en servicios intensivos en conocimiento, en particular. En la literatura se ha mostrado que el proceso de innovación en servicios es afectado por las características específicas de estos referidas a la inmaterialidad, la continua reconfiguración de la oferta y la simultaneidad entre provisión y consumo - conducentes a importantes interacciones con el cliente- que lo diferencian de la industria manufacturera. Por otra parte, la necesidad de comparación entre los procesos de innovación en industria y servicios, que sobrevino con el crecimiento de la relevancia de estos últimos, condujo a que se planteara un interesante debate en términos teóricos y metodológicos acerca de cómo abordar la problemática de la innovación en servicios. Este debate se desarrolló en torno de tres vertientes: i) una conceptualización

\footnotetext{
3 El concepto de clique alude a un conjunto de nodos que presenta todas las conexiones posibles ("todos conectados con todos"). Un cliqué puede tomar distintos órdenes en función de la cantidad de nodos que lo conforman; la díada y la tríada son las versiones más simples que pueden encontrarse en una red.
} 
"asimilacionista", ii) un criterio demarcatorio, y iii) una posición de síntesis.

El enfoque de asimilación fue apoyado por autores que planteaban que las diferencias intrasectoriales de las actividades innovativas eran mayores que las existentes entre industria y servicios en forma agregada (Hughes y Wood, 2000; Sirilli y Evangelista, 1998). Desde esta perspectiva, el estudio de los procesos de innovación en industria y servicios requiere de un tratamiento teórico y metodológico análogo.

En oposición a esta postura de asimilación, diversos autores (Gallouj y Weinstein, 1997; Djellal y Gallouj, 1999 y 2001; Coombs y Miles, 2000) avanzaron en el desarrollo de una visión demarcatoria, que atribuye especificidades a la innovación en servicios no aplicables a la industria manufacturera. Estos autores identificaron distintos tipos de innovación propios de servicios que en principio no podrían aplicarse a la actividad de la manufactura. Por ejemplo, las innovaciones que surgen de las interacciones con los clientes o aquellas que se refieren al proceso de codificación de los conocimientos tácitos generados en estas interacciones. En este contexto, por ejemplo, los desarrollos a medida darían lugar a innovaciones de producto de una forma prácticamente constante, sin que esto implique cambios radicales o incluso de incremento en las capacidades y rutinas de las firmas. Esta posición fue cuestionada por Drejer (2004) al plantear que en el enfoque demarcatorio se atribuyen especificidades a servicios que están presentes en la industria manufacturera. Entre ellas destaca: i) la importancia de las redes y de fuentes externas de conocimiento en la innovación (De Bresson y Amesse, 1991), ii) la centralidad del modelo interactivo y no lineal de innovación (Landau y Rosenberg, 1986), y iii) la relevancia de la codificación del conocimiento tácito.

Frente a estas críticas emerge una posición de síntesis cuya característica fundamental es reconocer atributos de servicios en la oferta de productos de la industria manufacturera y viceversa. Esto responde a la visión lancasteriana sobre la naturaleza de los productos y servicios, que fue retomada por Saviotti y Metcalfe (1984) y por Gallouj y Savona (2009). Dentro de esta posición, la innovación tiene lugar a partir de cambios en: i) las características del servicio desde la perspectiva del valor asignado por el usuario final, ii) las características técnicas materiales e inmateriales del producto, iii) las competencias de los oferentes, y iv) las competencias de los usuarios. En este contexto, las innovaciones de producto no responden al mero ajuste de la oferta a una nueva demanda, sino a los cambios en las competencias de oferentes y demandantes, y a cambios en las características percibidas del servicio (materiales e inmateriales) derivadas de esta demanda.

Considerando las discusiones de la literatura presentada, se parte de las siguientes premisas: i) los procesos innovativos de las firmas de ssi dependen del desarrollo de procesos de aprendizaje derivados de la retroalimentación entre capacidades y vinculaciones a lo largo de su sendero evolutivo, y ii) estos procesos se manifiestan en diferentes niveles de capacidades de absorción y organizacionales que repercuten de manera diferencial en los resultados de innovación.

De estas premisas surgen las siguientes hipótesis:

\section{Hipótesis 1}

Los resultados de innovación de las firmas de ssi argentinas dependen del desarrollo de capacidades organizacionales y de absorción.

Las interacciones entre empresas e instituciones configuran la arquitectura de conexiones que actúa como soporte para la circulación de la información y el conocimiento. En particular, es de esperar que las firmas ubicadas en posiciones intermedias en la red de conexiones muestren una mayor dinámica innovadora, ya que se nutren de conocimientos y capacidades tanto de las firmas que integran el núcleo de la red como de las ubicadas en posiciones periféricas. De esto último se desprende la segunda hipótesis.

\section{Hipótesis 2}

Los resultados de innovación de las firmas de sSI argentinas dependen de la posición que ocupan en la red de conexiones. En particular, son las posiciones intermedias las que se relacionan con una mayor probabilidad de obtener resultados de innovación.

Por otra parte, con la tercera hipótesis que se propone testear se pretende captar ciertas características de los procesos productivos discutidas en la literatura dedicada al estudio de la innovación en servicios, tales como la inmaterialidad o la innovación vinculada a la solución de problemas específicos planteados por los clientes. Estas características se manifiestan en diversos modelos de negocios de las firmas de ssi, definidos según la importancia de la relación proveedor-cliente, el nivel de estandarización del servicio y el grado de diversificación de los servicios ofertados por las firmas.

\section{Hipótesis 3}

Los resultados de innovación de las firmas de ssi argentinas están determinados por la micro-heterogeneidad dentro 
del sector, que se manifiesta en diferentes modelos de negocios. Es de esperar que las firmas en que los modelos de negocios predominantes son los desarrollos a medida y de productos propios tengan mayores resultados de innovación.

Finalmente, los enfoques teóricos discutidos permiten identificar diferentes determinantes de la innovación, en general, y en servicios, en particular. Sin embargo, queda pendiente relacionar estos determinantes (capacidades de absorción y organizacionales, posición de las firmas en la red y modelos de negocios) con el crecimiento reciente del sector manifestado en tres planos: el empleo, la productividad y las exportaciones.

\section{Hipótesis 4}

El desempeño de las firmas argentinas de ssi en términos de productividad, variación del empleo e inserción externa depende de: i) el nivel de desarrollo de capacidades organizacionales y de absorción, ii) la posición de las firmas en la red de conexiones, y iii) la micro-heterogeneidad manifestada en distintos modelos de negocios.

Con estas hipótesis se pretende estar más cerca de las preguntas de investigación planteadas en la Introducción, que se refieren a los determinantes de la innovación y del desempeño reciente del sector.

\section{III}

\section{Antecedentes empíricos sobre la estimación de innovación en SSI}

En los últimos años se desarrollaron diversos intentos con miras a conceptualizar y medir los determinantes de la innovación de firmas de ssi (Romijn y Albaladejo, 2002; Segelod y Jordan, 2002; Boschma y Weterings, 2005; Grimaldi y Torrisi, 2001; Parthasarathy y Aoyama, 2006; Corrocher, Cusmano y Morrison, 2009; Cusumano y Yoffie, 1999; Cusumano, 2012, entre otros). En términos generales, en estos trabajos se explora la relación entre desarrollo de capacidades, vinculaciones e innovación tanto en paneles de microdatos, como en estudios de caso. En los estudios se identifican algunas de las características clave del sector que hacen que los procesos de aprendizaje sean altamente específicos. Entre ellas destacan: i) el elevado peso del gasto en I+D sobre las ventas, ii) la importancia de la disponibilidad de recursos humanos altamente calificados en el total del personal, iii) la relevancia de las vinculaciones con universidades y centros tecnológicos, así como con firmas proveedoras; $y$ iv) la elevada frecuencia de desarrollos colaborativos entre clientes y proveedores en el marco de desarrollos a medida, contratación de servicios externos y comunidades de práctica, entre otros. Estas características repercuten de forma poco evidente en los resultados de innovación, e incluso ponen de manifiesto la necesidad de discutir la definición misma de innovación y sus formas de medición. De estos antecedentes, interesa aquí discutir tres trabajos que resultan centrales para esta investigación, puesto que abordan la cuestión de las interacciones, la relación con los clientes en el desarrollo de innovaciones y el análisis de los determinantes del desempeño de las firmas.

En primer término, Segelod y Jordan (2002) analizan el efecto de la relación proveedor-cliente en la introducción de innovaciones en 92 proyectos de software mayormente de Europa. Estos autores marcan la relevancia que adquieren las vinculaciones con clientes en los procesos de aprendizaje frente a las vinculaciones con centros tecnológicos y universidades. Además, muestran que el número de vínculos que tienen las empresas se relaciona positivamente con el nivel innovativo del proyecto y con la posibilidad de abrir nuevos mercados y por ende ampliar la competitividad de las firmas. Desde una perspectiva teórico-conceptual, estos autores destacan la importancia de la innovación focalizada en los usuarios (user-driven innovation) en el desarrollo de software y el acceso a nuevos mercados.

De acuerdo con estos autores, en el inicio del proceso de desarrollo de software los clientes de ssi tienen una percepción poco clara acerca del problema que desean solucionar, así como de las posibilidades tecnológicas y organizacionales potenciales que ese desarrollo ofrece. Por su parte, los desarrolladores tienen el conocimiento técnico, pero son ajenos al conocimiento respecto del área de aplicación del software. Por lo tanto, la definición de los requerimientos del software precisa de múltiples encuentros entre clientes y proveedores de soluciones de 
SSI, con el objetivo de reducir la brecha de conocimientos y realizar las traducciones necesarias en uno y otro sentido. Sin embargo, estas interacciones no se agotan en la etapa de diseño, ya que a medida que avanza el proyecto pueden surgir incompatibilidades técnicas que obliguen a su revisión. De tal forma, el proyecto parte de límites poco definidos que al cabo de un tiempo se irán delineando con más precisión aunque estarán sujetos a revisión a lo largo de todo su desarrollo, de modo que este tendrá un carácter inherentemente colaborativo. En este contexto, la implementación de un proyecto de software se relaciona más con un proyecto de I+D que con el desarrollo de un producto tangible. De acuerdo con lo planteado por Gallouj y Savona (2009), los procesos de retroalimentación con el cliente son claves en las fases de definición de los requerimientos, el diseño y el desarrollo.

En segundo lugar, Romijn y Albaladejo (2002) estudian los determinantes del desempeño innovativo de pequeñas firmas de ssi y electrónica en el Reino Unido de Gran Bretaña e Irlanda del Norte sobre la base de fuentes de conocimiento internas y externas. Las fuentes internas incluyen: i) el nivel educativo y la experiencia de los fundadores y gerentes, ii) la calificación profesional de la fuerza de trabajo, y iii) los esfuerzos tecnológicos como I+D formal e informal, capacitación formal e informal e inversiones en licencias tecnológicas. Las fuentes externas comprenden: i) la intensidad del establecimiento de redes (networking) con distintos agentes e instituciones, ii) las ventajas de la proximidad geográfica vinculadas al networking, y iii) el apoyo institucional. Estos autores se centran en la innovación de producto como medida de desempeño innovativo y utilizan tres indicadores: nuevos productos, número de patentes y un índice de innovación - construido sobre la base de información cualitativa - que da cuenta del alcance y la significatividad de los resultados de innovación de la firma. Dichos resultados muestran que entre las fuentes internas, la experiencia previa de los dueños y la proporción de ingenieros en la fuerza de trabajo producen un efecto positivo en el desempeño innovador de las firmas. También afirman que poseer conocimiento especializado y experiencia en ciencias e ingeniería constituye una condición previa para alcanzar resultados de innovación en firmas de alto nivel tecnológico. Sin embargo, ni la intensidad del networking ni la proximidad geográfica contribuyen al desempeño innovador. Solo las vinculaciones con instituciones de I+D y con proveedores tienen un efecto positivo.

Por último, Boschma y Weterings (2005) analizan 265 firmas holandesas de ssi y muestran que en las áreas geográficas con mayor desarrollo de las tecnologías de la información y las comunicaciones (TIC), la productividad, las oportunidades de negocios detectadas a partir de una empresa madre (spin-off) y las capacidades de las firmas son mayores. Estos autores consideran como variable dependiente la productividad innovativa, definida como el cociente entre las ventas de nuevos productos desarrollados por la empresa y el número de ocupados en I+D. Este indicador refleja la eficiencia de las rutinas y competencias de las firmas. Los autores muestran que el desarrollo de productos o servicios radicalmente nuevos repercute en forma positiva en la productividad innovativa. Asimismo, verifican que la participación de clientes y proveedores en el diseño del producto/servicio y la experiencia previa de la firma y de los dueños no tienen efectos en la productividad innovativa. Los resultados también ponen en evidencia que las empresas con menores dificultades para conseguir clientes tienen mayores capacidades tecno-organizacionales. Finalmente, consideran tres efectos conjuntos: i) tener experiencia previa en el sector de software incide positivamente en la relación entre el desarrollo de una innovación radical y una elevada productividad "innovativa"; ii) tener problemas para conseguir clientes requiere desarrollar un producto cuya innovación sea más radical, lo que se traduce en una disminución de su productividad, y iii) las firmas que presentan problemas para conseguir nuevos empleados tienen una mayor productividad en innovación cuando se localizan en regiones especializadas en TIC.

Finalmente, el antecedente más significativo de trabajos sobre innovación en ssi en la Argentina corresponde al último año de la convertibilidad con una muestra de 89 firmas y es de tipo descriptivo (Chudnovsky, López y Melitsko, 2001). En el trabajo se plantea que, si bien la mayor parte de las firmas lanzaron nuevos productos al mercado, las empresas nacionales - y en especial las orientadas a productos y no a serviciosfueron las más dinámicas en materia de innovación. Como plantean los autores, estos resultados deberían relativizarse debido a que el software es en sí mismo una actividad innovativa, lo que resalta la necesidad de desarrollar indicadores de innovación específicos para la actividad. 


\section{IV}

\section{Metodología, construcción de indicadores y estadística descriptiva}

La base de datos utilizada está constituida por 189 firmas de ssi entrevistadas en $2011^{4}$. En promedio, las firmas ocupan algo menos de 60 personas, tienen ventas anuales de alrededor de 1,8 millones de dólares y una activa participación en mercados externos. Se trata de empresas en que predomina el capital nacional, fundadas sobre todo durante la década de 1990. La mayor parte está localizada en la Capital Federal y el Gran Buenos Aires (74\%) y el resto se distribuye en las provincias de Córdoba (21\%) y Santa Fe (5\%). Entre 2008 y 2010 el número promedio de ocupados creció un $20 \%$, crecimiento muy superior al promedio de la economía (1\%).

Para estimar las capacidades internas de las firmas se construyó un conjunto de indicadores que reflejan dos dimensiones: capacidades de absorción y capacidades organizacionales. La capacidad de absorción fue estimada a partir de dos indicadores. El primero da cuenta del nivel de educación de los trabajadores y asume tres categorías ordinales: empresas con una proporción de universitarios menor que el promedio ${ }^{5} \mathrm{y}$ sin posgraduados (29\%); firmas con una proporción de profesionales universitarios mayor que el promedio y sin posgraduados (26\%), y empresas con posgraduados más allá del peso de profesionales universitarios en la ocupación total (45\%). El segundo indicador refleja la disponibilidad y el grado de formalidad del equipo de

\footnotetext{
${ }^{4}$ El diseño muestral se basó en un trabajo previo de caracterización del universo realizado en conjunto con el Observatorio de Empleo y Dinámica Empresarial del Ministerio de Trabajo, Empleo y Seguridad Social. En la muestra se consideraron dos planos: el tamaño de las firmas y la incidencia de los subsidios públicos otorgados por el Ministerio de la Producción a través de la Ley de Promoción de la Industria del Software, y por el Ministerio de Ciencia, Tecnología e Innovación Productiva a través del Fondo Tecnológico Argentino (FONTAR) y el Fondo Fiduciario de Promoción de la Industria del Software (FONSOFT). En 2010 el sector contaba con alrededor de 1.600 firmas de 5 y más ocupados que daban empleo a cerca de 57.000 trabajadores. Las firmas de menos de cinco ocupados mostraron una baja tasa de permanencia en el mercado, motivo por el cual fueron excluidas del análisis del universo y del diseño muestral. Por su parte, las empresas beneficiadas con programas públicos alcanzaban al $22 \%$ del universo de cinco y más ocupados.

${ }^{5}$ El promedio de los universitarios (40\%) es significativamente elevado cuando se lo compara con el promedio de la industria manufacturera (13\%); véase la Encuesta Nacional de Innovación Tecnológica (ENIT) del Instituto Nacional de Estadística y Censos (INDEC), 2005.
}

I+D. La variable contempla cuatro categorías: no tiene equipo (11\%), equipo informal esporádico (32\%), equipo informal regular (28\%) y equipo formal (29\%). Las capacidades organizacionales se estiman sobre la base de: i) una variable aditiva en que se consideran los esfuerzos de las firmas en gestión de calidad ${ }^{6}$ y que asume tres valores posibles: uno para las firmas que realizaron hasta 1 esfuerzo ( $25 \%$ de las firmas), dos para las firmas que realizaron entre 2 y 3 esfuerzos $(37 \%)$ y tres para las firmas que realizaron 4 ó 5 esfuerzos, ii) una variable que da cuenta de la cantidad de certificaciones de calidad, entre las que se incluyen las normas Iso 9000, el modelo de madurez de capacidades (CMM), TickIT $^{7}$ y SLA (Service Level Agreement), que asume tres valores posibles: uno para las firmas sin certificación de calidad (39\%), dos para las firmas con una certificación (43\%) y tres para las firmas con dos y más certificaciones (18\%), y iii) una variable que da cuenta del uso de metodologías ágiles ${ }^{8}$ y asume tres valores si las empresas no usan nunca dichas metodologías (18\%), las usan a veces (44\%) o las utilizan siempre (38\%). En el cuadro 1 se presenta la matriz de correlaciones de los indicadores de capacidades internas, que permite identificar algunas relaciones estadísticamente significativas. La presencia y formalidad del equipo de I+D muestra una relación positiva con la gestión de la calidad. Por su parte, el grado de calificación de los trabajadores muestra una correlación positiva con la certificación de calidad.

\footnotetext{
6 Se consideraron cinco alternativas que diferenciaban la gestión de calidad de las firmas: trazabilidad, auditorías internas, gestión de riesgos, disponibilidad de personal dedicado a la gestión de calidad y existencia de área formal de calidad.

7 Programa de certificación de administración de la calidad para el software, apoyado sobre todo por el Reino Unido de Gran Bretaña e Irlanda del Norte y las industrias suecas de software.

${ }^{8}$ Las metodologías ágiles son una nueva forma de organización del trabajo del sector de ssi. Se trata de un método basado en el desarrollo iterativo e incremental, donde los requerimientos y soluciones evolucionan a partir de la colaboración continua con los clientes y diferentes grupos de trabajo. Este método es principalmente funcional a la subcontratación externa (outsourcing) y a los desarrollos a medida, ya que favorece el control de la evolución de los proyectos a lo largo de su ejecución.
} 
Matriz de correlaciones entre los indicadores de capacidades (Coeficientes de correlación)

\begin{tabular}{|c|c|c|c|c|c|c|}
\hline & & \multicolumn{2}{|c|}{ Capacidades de absorción } & \multicolumn{3}{|c|}{ Capacidades organizacionales } \\
\hline & & Equipo I+D & $\begin{array}{l}\text { Calificación de } \\
\text { los trabajadores }\end{array}$ & $\begin{array}{l}\text { Metodologías } \\
\text { ágiles }\end{array}$ & Calidad & Certificación \\
\hline \multirow[b]{2}{*}{$\begin{array}{l}\text { Capacidades de } \\
\text { absorción }\end{array}$} & Equipo I+D & 1 & & & & \\
\hline & $\begin{array}{l}\text { Calificación de los } \\
\text { trabajadores }\end{array}$ & $0,2005^{*}$ & 1 & & & \\
\hline \multirow{3}{*}{$\begin{array}{l}\text { Capacidades } \\
\text { organizacionales }\end{array}$} & Metodologías ágiles & 0,1132 & 0,0992 & 1 & & \\
\hline & Calidad & $0,3152 *$ & $0,2179 *$ & 0,1055 & 1 & \\
\hline & Certificación & 0,0976 & $0,1823 *$ & 0,1041 & $0,4943^{*}$ & 1 \\
\hline
\end{tabular}

Fuente: elaboración propia sobre la base de una encuesta realizada en el marco del proyecto financiado por la Fundación Carolina.

*Significativo al $10 \%$.

Nota: los coeficientes sin asterisco no son estadísticamente significativos.

Las variables estructurales consideradas son el tamaño de la firma — estimado por el número de ocupados-, su localización geográfica, su antigüedad y el acceso que tuvo a instrumentos de promoción ${ }^{9}$. Las relaciones entre los indicadores de capacidades y las variables estructurales se presentan en el anexo A. Entre los resultados significativos de los cuadros de contingencia surgen las siguientes correlaciones: i) todos los indicadores de capacidades se relacionan con el tamaño de la firma; ii) la antigüedad de las firmas solo se relaciona positivamente con el indicador de calidad, lo que significa que las firmas más antiguas están sobrerrepresentadas entre las que realizan mayores esfuerzos de calidad; iii) la presencia de subsidios muestra una relación positiva con los indicadores de gestión de calidad y certificaciones, dado que en general los programas de promoción apoyan este tipo de actividades, iv) finalmente, no existe vinculación entre los indicadores de capacidades y la localización geográfica de las firmas.

Para evaluar el nivel de conectividad de las firmas se construyó la red de conocimiento que involucra a empresas e instituciones de fomento a la innovación. En esta red se consideran las vinculaciones que las firmas mantienen con otras empresas e instituciones - como universidades, centros tecnológicos y consultoresorientadas hacia actividades de I+D, calidad y asistencia técnica (véase el anexo B). Para estimar el nivel de conectividad de las firmas se partió de su posición en

${ }^{9}$ Las firmas promocionadas son aquellas que participan de la Ley de Software y reciben ayuda del FONTAR o del FONSOFT pertenecientes al Ministerio de Ciencia, Tecnología e Innovación Productiva. la red de conexiones y se calculó el coreness $^{10}$, que constituye una medida de centralidad indicadora del grado de pertenencia al núcleo central en una estructura centro-periferia. Este indicador permite establecer un orden jerárquico ${ }^{11}$. De esta manera, las firmas periféricas reciben un bajo coeficiente de coreness, mientras que a las que ocupan un papel central se les asigna un valor más alto, más allá del número de conexiones o grado (degree) que tengan. En el cuadro 2 se muestra que existe una relación positiva entre el tamaño y el coreness y que las firmas localizadas en Buenos Aires y Córdoba tienen un coreness mayor que las ubicadas en Santa Fe. Por el contrario, no se observan diferencias significativas al considerar la antigüedad y los subsidios recibidos.

10 Cercanía de cada nodo al conjunto de nodos densamente interconectados entre sí que presenta la red. Para calcular el coreness se utilizó el algoritmo de núcleo/periferia en su versión continua disponible en el software UCINET (Borgatti, Everett y Freeman, 2002). El algoritmo es el siguiente:

$$
\rho=\sum_{i, j} a_{i, j} \delta_{i, j} \quad \text { donde } \delta_{\mathrm{i}, \mathrm{j}}=\begin{aligned}
& 1 \text { si está en el núcleo y } \\
& 0 \text { en el caso contrario. }
\end{aligned}
$$

Se optó por el coreness, y no por otras medidas de centralidad — como el grado (degree), la intermediación (betweeness) y la cercanía (closeness) porque este estadístico de red permite resaltar la complementariedad entre los aspectos de densidad y agujeros estructurales (structural holes). Esto es, una mayor densidad de lazos directos puede no ser beneficiosa para la firma, puesto que no aporta necesariamente nueva información, a menos que los lazos permitan conectar nodos que de otra forma permanecerían aislados.

11 Esto va más allá de las estimaciones realizadas por Romijn y Albaladejo (2002) y por Segelod y Jordan (2002), que analizan la conectividad desde una perspectiva egocéntrica (es decir, que parte de las conexiones que se pueden trazar a partir de un ego/nodo dado) similar a lo que sería el indicador de grado (degree) en las redes sociales. 


\section{Relación entre la coreness y las variables estructurales}

\begin{tabular}{lc|lc|lc|cc}
\hline Tamaño & Coreness & Antigüedad & Coreness & Localización & Coreness & Subsidio & Coreness \\
\hline Hasta 10 empleados & 0,004 & Hasta 1990 & 0,016 & Buenos Aires & 0,016 & No & 0,015 \\
De 11 a 50 empleados & 0,016 & 1991 a 2001 & 0,020 & Córdoba & 0,014 & Sí & 0,016 \\
51 y más empleados & 0,028 & 2002 a 2005 & 0,009 & Santa Fe & 0,004 & & \\
& & 2006 en adelante & 0,014 & & & & \\
\hline
\end{tabular}

Fuente: elaboración propia sobre la base de un levantamiento realizado en el marco de un proyecto financiado por la Fundación Carolina.

Coreness: coeficiente que mide la centralidad de la firma en la red de conexiones.

Por otra parte, las firmas fueron consultadas sobre la obtención de resultados de innovación durante el período 2008-2010, considerando seis alternativas: i) nuevos productos; ii) nuevos servicios; iii) productos con mejoras significativas; iv) procesos nuevos o con mejoras significativas; v) nuevos canales de comercialización, y vi) cambios organizacionales. A partir de estas alternativas, se construyó un indicador que toma tres valores posibles: vale 1 en el caso de las firmas que obtuvieron hasta una innovación ${ }^{12}, 2$ para las firmas que desarrollaron de 2 a 4 innovaciones y 3 para las firmas con 5 y 6 resultados de innovación. La mayoría de las empresas (58\%) se encuentran en el segundo grupo, mientras que los grupos primero y tercero están compuestos por el $24 \%$ y el $19 \%$ de las firmas, respectivamente.

Para evaluar el desempeño de las firmas se construyeron tres indicadores: inserción externa en 2010, variación del empleo entre 2008 y 2010 y productividad del trabajo en 2010. La "inserción externa" de las firmas se estima a partir de una variable binaria que indica si las firmas exportaron o no en 2010. En el indicador de "variación del empleo" entre 2008 y 2010 se consideran cuatro categorías: i) firmas en que disminuyó el empleo (19\%); ii) firmas en que el empleo se mantuvo constante (19\%); iii) firmas en que aumentó el empleo hasta el $45 \%$ (33\%), y iv) firmas cuyo empleo creció más del $45 \%$ durante el período (29\%). Este indicador se relaciona positivamente con el tamaño de la empresa, lo que denota la presencia de un proceso de concentración durante el período.

La "productividad del trabajo" se estimó sobre la base de la facturación por ocupado durante el año 2010. Esta variable continua fue codificada en cuatro tramos

\footnotetext{
12 Dado que la mayoría de las firmas obtuvieron innovaciones (90\%), el grupo de control se formó con aquellas que no innovaron y con las que obtuvieron solo un resultado.
}

a partir de la medida de cuartiles de la distribución de ventas por ocupado. Se vincula positivamente con la antigüedad de las firmas.

La micro-heterogeneidad del sector se refleja en distintos modelos de negocios adoptados por las firmas: i) desarrollos a medida; ii) desarrollos y servicios sobre productos propios; iii) subcontratación externa (outsourcing); iv) servicios sobre desarrollos de terceros, y v) modelo diversificado. El cruce con las variables estructurales (véase el cuadro 3) revela que entre las firmas que reciben subsidios se destacan aquellas que desarrollan productos a medida o siguen un modelo de negocios más diversificado. Mientras que entre las firmas que tienen entre 11 y 50 ocupados predominan las que desarrollan y brindan servicios sobre productos propios, en el grupo con más de 50 empleados destacan las que realizan productos a medida. Por otra parte, las firmas creadas entre 1991 y 2001 muestran una orientación más diversificada, en tanto que las más nuevas se dedican a la subcontratación externa (outsourcing) u ofrecen servicios relacionados con desarrollos de terceros. Finalmente, en cuanto a la localización geográfica, las firmas de Buenos Aires son las que siguen un modelo de negocios más diversificado, las de Córdoba muestran una orientación predominante hacia el desarrollo de productos a medida y las de Santa Fe al desarrollo y servicios de productos propios.

Finalmente se analiza la relación entre las capacidades y los diversos modelos de negocios (véase el cuadro 4). El grupo de firmas que realizan productos a medida se destaca por tener un equipo formal de I+D, la presencia de posgraduados y la mayor cantidad de esfuerzos de calidad y certificaciones. Esto refleja que las firmas que enfrentan demandas específicas de sus clientes muestran el umbral más alto de capacidades. Asimismo, en el modelo de negocios orientado a la subcontratación externa (outsourcing) se destaca la disponibilidad de certificaciones de calidad y la ausencia de equipo de I+D. 
CUADRO 3

Relación entre los modelos de negocios y las variables estructurales (En porcentajes)

Clasificación de los modelos de negocios

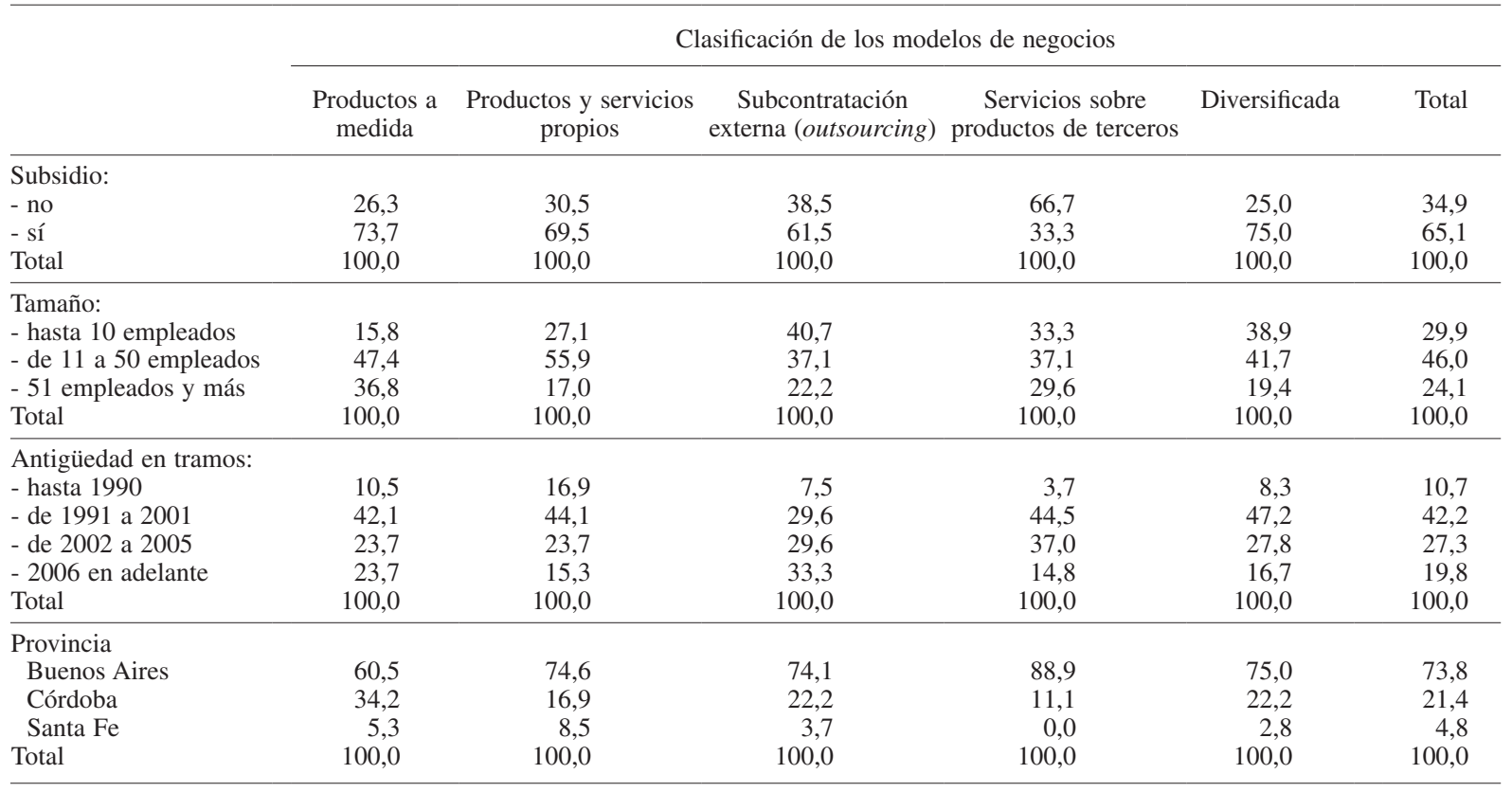

Fuente: elaboración propia sobre la base de un levantamiento realizado en el marco de un proyecto financiado por la Fundación Carolina.

CUADRO 4

Relación entre los modelos de negocios y los indicadores de capacidades (En porcentajes)

\begin{tabular}{|c|c|c|c|c|c|c|}
\hline & & & Clasificación de los me & odelos de negocios & & \\
\hline & $\begin{array}{l}\text { Productos } \\
\text { a medida }\end{array}$ & $\begin{array}{c}\text { Productos y } \\
\text { servicios propios }\end{array}$ & $\begin{array}{l}\text { Subcontratación } \\
\text { externa (outsourcing) }\end{array}$ & $\begin{array}{l}\text { Servicios sobre } \\
\text { productos de terceros }\end{array}$ & Diversificada & Total \\
\hline $\begin{array}{l}\text { Presencia de equipo de I+D: } \\
\text { - no tiene } \\
\text { - informal esporádico } \\
\text { - informal regular } \\
\text { - formal } \\
\text { Total }\end{array}$ & $\begin{array}{r}0,1 \\
36,8 \\
18,4 \\
44,7 \\
100,0\end{array}$ & $\begin{array}{r}1,7 \\
31,0 \\
34,5 \\
32,8 \\
100,0\end{array}$ & $\begin{array}{r}37,1 \\
33,3 \\
22,2 \\
7,4 \\
100,0\end{array}$ & $\begin{array}{r}14,8 \\
33,4 \\
25,9 \\
25,9 \\
100,0\end{array}$ & $\begin{array}{r}13,9 \\
30,6 \\
30,5 \\
25,0 \\
100,0\end{array}$ & $\begin{array}{r}10,8 \\
32,8 \\
27,4 \\
29,0 \\
100,0\end{array}$ \\
\hline $\begin{array}{l}\text { Capacidades de absorción: } \\
\text { - baja } \\
\text { - media } \\
\text { - alta } \\
\text { Total }\end{array}$ & $\begin{array}{r}28,9 \\
18,5 \\
52,6 \\
100,0\end{array}$ & $\begin{array}{r}33,9 \\
25,0 \\
41,1 \\
100,0\end{array}$ & $\begin{array}{r}23,1 \\
34,6 \\
42,3 \\
100,0\end{array}$ & $\begin{array}{r}19,2 \\
23,1 \\
57,7 \\
100,0\end{array}$ & $\begin{array}{r}33,3 \\
27,8 \\
38,9 \\
100,0\end{array}$ & $\begin{array}{r}29,1 \\
25,3 \\
45,6 \\
100,0\end{array}$ \\
\hline $\begin{array}{l}\text { Esfuerzos de calidad: } \\
\text { - hasta } 1 \text { esfuerzo } \\
\text { - entre } 2 \text { y } 3 \text { esfuerzos } \\
\text { - } 4 \text { ó } 5 \text { esfuerzos } \\
\text { Total }\end{array}$ & $\begin{array}{r}21,1 \\
26,3 \\
52,6 \\
100,0\end{array}$ & $\begin{array}{r}24,1 \\
31,1 \\
44,8 \\
100,0\end{array}$ & $\begin{array}{r}16,7 \\
45,8 \\
37,5 \\
100,0\end{array}$ & $\begin{array}{r}37,1 \\
44,4 \\
18,5 \\
100,0\end{array}$ & $\begin{array}{r}27,8 \\
44,4 \\
27,8 \\
100,0\end{array}$ & $\begin{array}{r}25,1 \\
36,6 \\
38,3 \\
100,0\end{array}$ \\
\hline $\begin{array}{l}\text { Cantidad de certificaciones: } \\
\text { - no tiene } \\
\text { - tiene una certificación } \\
\text { - tiene dos y más certificaciones } \\
\text { Total }\end{array}$ & $\begin{array}{r}34,2 \\
42,1 \\
23,7 \\
100,0\end{array}$ & $\begin{array}{r}39,6 \\
48,3 \\
12,1 \\
100,0\end{array}$ & $\begin{array}{r}29,2 \\
45,8 \\
25,0 \\
100,0\end{array}$ & $\begin{array}{r}42,3 \\
42,3 \\
15,4 \\
100,0\end{array}$ & $\begin{array}{r}47,2 \\
33,3 \\
19,5 \\
100,0\end{array}$ & $\begin{array}{r}39,0 \\
42,9 \\
18,1 \\
100,0\end{array}$ \\
\hline
\end{tabular}

Fuente: elaboración propia sobre la base de un levantamiento realizado en el marco de un proyecto financiado por la Fundación Carolina. I+D: investigación y desarrrollo. 


\section{V}

\section{Principales resultados de los modelos estimados}

En esta sección se presenta un conjunto de modelos econométricos estimados para testear el efecto del desarrollo de capacidades —organizacionales y de absorción-, de la ubicación de las firmas en la red de conexiones y de los modelos de negocios en la conducta innovadora y el desempeño de las firmas. El modelo propuesto es el siguiente:

$$
\begin{aligned}
& Y_{i}=f\left(c a p \_a b s o r c i o ́ n, ; c a p \_t e c n o l o ́ g i c a_{i} ;\right. \\
& \text { cap_organizacionales }_{i} ; \text { modelo_negocios }_{i} \text {; } \\
& \text { posición_red }_{i} \text {; } \text { vbles_control }_{i} \text {; término_error }{ }_{i} \text { ) }
\end{aligned}
$$

A partir de esta ecuación se estimaron cuatro modelos. En los casos en que la variable dependiente fuera discreta y ordinal (innovación, productividad del trabajo y variación del empleo) se utilizó un modelo probabilístico para datos ordenados. A su vez, para explicar la inserción externa de las firmas se recurrió a un modelo probabilístico. Entre las variables independientes, se utilizaron: i) los indicadores de capacidades (cap absorción, cap_tecnológica y cap_organizacionales de la ecuación); ii) el coreness que registra cada firma en la red de vinculaciones y el coreness al cuadrado (posición_red) para considerar la posibilidad de una relación cuadrática entre este indicador, la innovación y las medidas de desempeño, y iii) el modelo de negocios (modelo_negocios), para lo cual se consideraron cuatro variables ficticias quedando como categoría base el modelo de negocios diversificado. Por último, como variables de control (vbles_control) se incluyeron la disponibilidad de subsidios, el tamaño de las firmas, su antigüedad y su localización. Con el primer modelo se estiman los determinantes de la cantidad de innovaciones desarrolladas por las empresas. Confirmando parcialmente la primera hipótesis formulada en este trabajo, la disponibilidad y el grado de formalidad del equipo de I+D resultaron significativos, mostrando la relevancia de las capacidades de absorción en el desarrollo de innovaciones ${ }^{13}$. Entre las capacidades organizacionales resultó significativa la gestión de calidad. En concordancia con la hipótesis 2, la posición de la firma en la red de

\footnotetext{
13 Un resultado similar fue encontrado por Romijn y Albaladejo (2002).
}

conexiones incide positivamente en la cantidad de innovaciones. En particular, tanto el coreness (centralidad) como el coreness al cuadrado son estadísticamente significativos, con signo positivo en el primer caso y negativo en el segundo. Este resultado indica que a medida que aumenta la centralidad de las firmas en la red, se incrementa la probabilidad de obtener mayor cantidad de resultados de innovación, pero a partir de cierto umbral dicha probabilidad comienza a descender. Esto sugiere que las posiciones intermedias en la red dan lugar a que las firmas se nutran de los conocimientos y capacidades tanto de otras más periféricas, como de las que forman parte del núcleo. En términos de la hipótesis 3 , el indicador que recoge las diferentes clasificaciones del modelo de negocios señala que el grupo de firmas que realizan servicios sobre productos de terceros, o efectúan subcontratación externa (outsourcing), alcanzan en promedio menores resultados de innovación que aquellas con una estrategia de negocios diversificada. Estos resultados no corroboran la hipótesis planteada, ya que el desarrollo a medida y de productos y servicios propios no repercute mayormente en los resultados de innovación en relación con la estrategia diversificada.

En el segundo modelo se analizan los determinantes del nivel de productividad de las firmas. En este caso, solo la capacidad de absorción, estimada a partir de la calificación de los recursos humanos, afecta positivamente. Es decir, la productividad de la firma se incrementa en la medida en que la proporción de profesionales supera a la media del sector, pero aumenta aún más para el conjunto de firmas que -independientemente del peso de los profesionales universitarios en la ocupación - tienen posgraduados. Por último, del conjunto de variables de control propuestas solo aparece como significativa la antigüedad de la firma. O sea, las firmas más antiguas son las de mayor productividad.

Cabe hace notar que ninguna de las otras variables con que se estiman las capacidades, las vinculaciones y los distintos modelos de negocios son significativas para explicar la productividad. Este resultado puede deberse a que en muchos casos las firmas expanden su empleo sin colocar a los trabajadores en proyectos específicos. Esto ocurre en un contexto en que la cantidad y calidad de recursos humanos son una restricción significativa 
en el sector y las firmas tienen que realizar importantes esfuerzos de capacitación interna para adaptar los recursos humanos existentes. Pero también se explica en parte debido a que dentro del conjunto de firmas de baja productividad, conviven firmas con altas y bajas capacidades y por consiguiente no es posible encontrar relaciones significativas en el modelo. Este resultado también da cuenta de las características de la competencia en el sector, donde su alto crecimiento alienta la posibilidad de sostener bajos niveles de productividad en general, con independencia de las capacidades de las firmas y de su evolución futura. Es decir, en términos de una explicación schumpeteriana de destrucción creativa, se puede afirmar que el sector se encuentra en la etapa de generación de variedad más que en la etapa de su resolución a través de la selección.

Los determinantes de la variación del empleo son abordados en el tercer modelo. Sus resultados indican que del conjunto de variables independientes propuestas, solo es significativa la capacidad de absorción, estimada a partir de la disponibilidad y el grado de formalidad del equipo de I+D. Es decir, pasar del grupo de firmas que no cuentan con equipo de I+D al de firmas que cuentan con equipo informal y al de aquellas que disponen de departamentos formales de I+D se relaciona con una mayor tasa de variación del empleo. En este caso, ni los modelos de negocios ni la posición de la firma en la red resultan significativos para explicar la variación en el empleo.

Entre las variables de control, el tamaño de las firmas se vincula positivamente al incremento del empleo. Este resultado sugiere que las firmas más grandes son las que más aumentaron el empleo. Esto se halla en línea con la tendencia a una mayor concentración del sector de sSI observada en los años recientes (OEDE/ MTSS, 2012). Asimismo, se constata una relación positiva y significativa entre la antigüedad de las firmas y la variación del empleo, lo que indica que son las firmas más nuevas las que presentan una mayor probabilidad de incrementar el número de sus ocupados.
Por su parte, el cuarto modelo muestra que la probabilidad de insertarse en mercados externos se relaciona positivamente con la centralidad de las firmas en la red y negativamente con el indicador de coreness al cuadrado. La lectura de estos resultados es similar a la realizada anteriormente y refleja que las firmas ubicadas en posiciones intermedias en la arquitectura de red tienen mayor probabilidad de exportar. A su vez, solo las capacidades organizacionales están vinculadas a la existencia de exportaciones. En particular, destaca la presencia de certificaciones y la utilización de metodologías ágiles. Estos resultados se explican tanto por la necesidad de cumplir con estándares internacionales para acceder a los mercados externos, como por la relevancia de las metodologías ágiles que dan cuenta de las necesidades de revisión y control que conllevan los desarrollos para mercados externos. Dichos resultados también ponen de manifiesto que, a diferencia de lo que suele encontrarse respecto de gran parte de la industria manufacturera, el tipo de inserción externa predominante no requiere necesariamente de un elevado nivel de desarrollo de capacidades internas.

Finalmente, dando cuenta del papel desempeñado por la micro-heterogeneidad de modelos de negocios, las firmas que realizan servicios relativos a desarrollos propios presentan una probabilidad de exportar mayor en comparación con las que se caracterizan por tener una estrategia diversificada. Entre las variables de control, los resultados muestran que el tamaño de las firmas no es significativo, lo que - a diferencia de lo que sucede en la industria manufacturera- se explica por la ausencia de elevados costos de logística vinculados a la exportación de ssi.

En suma, del análisis de los últimos tres modelos surge que la hipótesis 4 se cumple de manera parcial, con distintos resultados según la medida de desempeño considerada. En consecuencia, la heterogeneidad de estos resultados evidencia la imposibilidad de explicar el desempeño de cada firma a partir de un conjunto homogéneo de indicadores. 


\begin{tabular}{|c|c|c|c|c|c|}
\hline & \multirow{3}{*}{$\begin{array}{c}\text { Variables } \\
\text { independientes }\end{array}$} & \multicolumn{4}{|c|}{ Variables dependientes: indicadores de desempeño } \\
\hline & & Innovación (1) & Productividad (2) & Empleo (3) & Exportación (4) \\
\hline & & $\begin{array}{l}\text { Probit para datos } \\
\text { ordenados }\end{array}$ & $\begin{array}{l}\text { Probit para datos } \\
\text { ordenados }\end{array}$ & $\begin{array}{l}\text { Probit para datos } \\
\text { ordenados }\end{array}$ & $\begin{array}{c}\text { Probit para datos } \\
\text { ordenados }\end{array}$ \\
\hline \multirow{2}{*}{$\begin{array}{l}\text { Posición en } \\
\text { la red }\end{array}$} & Coreness & $9,716^{*}$ & 7,099 & 1,239 & $44,550 * * *$ \\
\hline & Coreness al cuadrado & $-36,883 *$ & $-11,38$ & $-5,22$ & $-318,693 * *$ \\
\hline \multirow{2}{*}{$\begin{array}{l}\text { Capacidades } \\
\text { de absorción }\end{array}$} & Equipo de I\&D & $0,200 *$ & 0,111 & $0,239 * *$ & 0,027 \\
\hline & $\begin{array}{l}\text { Calificación de los } \\
\text { recursos humanos }\end{array}$ & 0,096 & $0,260 * *$ & $-0,024$ & 0,206 \\
\hline \multirow{3}{*}{$\begin{array}{l}\text { Capacidades } \\
\text { organizacionales }\end{array}$} & Gestión de calidad & $0,277 *$ & 0,037 & $-0,099$ & $-0,094$ \\
\hline & Certificaciones de calidad & $-0,145$ & $-0,021$ & $-0,081$ & $0,590 * * *$ \\
\hline & Metodologías ágiles & 0,115 & 0,008 & $-0,041$ & $0,421 * * *$ \\
\hline \multirow{4}{*}{$\begin{array}{l}\text { Modelos de } \\
\text { negocios }\end{array}$} & Desarrollos a medida & $-0,036$ & 0,285 & $-0,042$ & 0,427 \\
\hline & $\begin{array}{l}\text { Productos y servicios } \\
\text { propios }\end{array}$ & $-0,212$ & 0,348 & $-0,244$ & $0,724 * *$ \\
\hline & $\begin{array}{l}\text { Servicios sobre desarrollos } \\
\text { de terceros }\end{array}$ & $-0,562 *$ & 0,245 & $-0,226$ & 0,085 \\
\hline & $\begin{array}{l}\text { Subcontratación externa } \\
\text { (outsourcing) }\end{array}$ & $-0,629 *$ & 0,071 & $-0,086$ & 0,627 \\
\hline \multirow{6}{*}{$\begin{array}{l}\text { Variables de } \\
\text { control }\end{array}$} & Apoyo público & $-0,006$ & $-0,028$ & 0,163 & 0,337 \\
\hline & Tamaño & $-0,134$ & $-0,289$ & $0,418 * *$ & $-0,059$ \\
\hline & Antigüedad & 0,006 & $-0,303 * * *$ & 0,394 *** & 0,153 \\
\hline & Localización & 0,041 & $-0,118$ & $-0,1$ & 0,269 \\
\hline & Constante & $* * *$ & *** & *** & $* * *$ \\
\hline & $\mathrm{N}^{\mathrm{o}}$ de observaciones & 189 & 189 & 189 & 189 \\
\hline
\end{tabular}

Fuente: elaboración propia sobre una base de datos construida en el marco del proyecto "Capacidades de absorción y conectividad en sistemas productivos y de innovación locales" financiado por la Fundación Carolina.

Nota: *significativo al 10\%; **significativo al 5\%; ***significativo al $1 \%$.

\section{VI}

\section{Conclusiones}

En esta investigación se estudiaron los procesos de innovación y el desempeño de firmas de ssi argentinas a partir de las capacidades organizacionales y de absorción que fueron construyendo a lo largo de su sendero evolutivo, y del lugar que ocupan en la arquitectura de conexiones. En esta dirección, se procuró dar una explicación evolutiva del crecimiento reciente del sector, en que se considera la construcción de capacidades y la articulación de conocimientos externos e internos. Por otra parte, las diferencias de las firmas en torno de distintos modelos de negocios indican que se trata de un sector heterogéneo y que el desarrollo desigual de capacidades responde a las necesidades específicas de cada subsegmento y tiene una repercusión diferencial en términos de empleo, productividad y exportaciones.

Se logró incorporar en el análisis algunas dimensiones clave para entender las capacidades de las firmas y sus vinculaciones con otros agentes e instituciones orientados al mejoramiento de sus capacidades, que se acercan en parte a las propuestas de Gallouj y Savona (2009). En tal sentido, se diseñaron indicadores de las capacidades organizacionales y de absorción que, dado que se trata de un sector intensivo en conocimiento, van más allá de los utilizados tradicionalmente en las encuestas de innovación. Por una parte, en el caso de las capacidades organizacionales se incluyeron el uso de metodologías 
ágiles, la gestión de calidad y la implementación de normas de calidad específicas. Por otra, las capacidades de absorción fueron estimadas a partir: i) del peso de profesionales y la presencia de posgraduados en el plantel de ocupados de la firma, y ii) de la presencia y formalidad de los equipos de I+D en un indicador en que se considera un gradiente de situaciones que van desde la ausencia de un grupo de resolución de problemas, hasta la presencia de un grupo formal de I+D. Esto es especialmente relevante en este sector en que la escala no es dominante, sobre todo en algunos modelos de negocios como el basado en desarrollos a medida.

Los resultados de los modelos permiten verificar en parte las tres primeras hipótesis. Los resultados de innovación dependen de la gestión de la calidad, del equipo de I+D y de tener una ubicación intermedia en la red de conexiones. En relación con la cuarta hipótesis, los modelos revelan que las diferentes medidas de desempeño en el sector de ssi no pueden explicarse por el mismo conjunto de indicadores. Las diferencias entre firmas en el interior del sector no solo en términos de capacidades y conectividad, sino también de desempeño, constituyen un rasgo que no puede ignorarse. Mientras que el nivel de productividad del trabajo se explica por la calificación de los trabajadores, la dinámica del empleo se relaciona positivamente con la existencia y formalidad del equipo de I+D y el tamaño de las firmas, en tanto que la inserción externa depende de la certificación de calidad, el uso de metodologías agiles y una posición intermedia en la arquitectura de la red.

En el trabajo resaltan las dificultades teóricas y metodológicas para abordar los procesos de innovación en sectores de ssi intensivos en conocimiento, dado que la estimación de la actividad de innovación a partir de metodologías utilizadas en la industria manufacturera - centradas en el desarrollo de nuevos productos y procesos- pierde poder explicativo. Esto se agrava aún más en los casos de firmas que efectúan desarrollos a medida, ya que el desarrollo de un nuevo producto o proceso es la naturaleza de su actividad y no constituye necesariamente una innovación. En ese sentido, tanto en la discusión de la literatura presentada en el trabajo, como en la discusión de la estadística descriptiva y del modelo, se dejan planteados los problemas que debería incluir una agenda de investigación sobre la relación entre capacidades, esfuerzos de innovación y desempeño en un sector como el abordado en esta investigación, que tiene una creciente importancia en la estructura ocupacional de la región. En concordancia con lo planteado por Gallouj y Savona (2009), el análisis de los resultados de este estudio conduce a la necesidad de elaborar un enfoque en que se consideren las especificidades del sector de ssi y que, a su vez, se tomen en cuenta aquellas dimensiones del proceso de innovación de la industria manufacturera que son asimilables al desarrollo de ssi (Drejer, 2004). Entre ellas, es sobre todo importante estudiar los procesos de innovación como fenómenos interactivos o sistémicos, en los que son clave la vinculación cliente-proveedor, la presencia en redes y el uso de fuentes externas de conocimiento.

Esto abre nuevas preguntas y líneas futuras de investigación centradas en la necesidad de: i) avanzar en una conceptualización de innovación para servicios en general y de ssi, y ii) definir cuáles son las medidas que dan cuenta del éxito de las firmas de ssi en el mercado. Esto requeriría, además, estudiar qué tipo de trayectorias tecnológicas y senderos evolutivos de las firmas se vinculan a un buen desempeño y cómo se manifiestan los mecanismos de retroalimentación entre conectividad y capacidades tecnológicas y organizacionales que pueden dar lugar a la innovación como propiedad emergente. Este conjunto de dimensiones requeriría desarrollar estudios de caso en firmas de muy diferentes capacidades tecnológicas, organizacionales, comerciales y de absorción, y de muy desiguales modelos de negocios en que se especializan y posición en la arquitectura de red. 
ANEXO A

CUADRO A 1

Relación entre variables de capacidades y estructurales

(En porcentajes)

\begin{tabular}{|c|c|c|c|c|}
\hline & \multicolumn{4}{|c|}{ Tamaño en tramos, año 2010} \\
\hline & Hasta 10 & De 11 a 50 & 51 y más & Total \\
\hline \multicolumn{5}{|l|}{ Equipo de I+D: } \\
\hline - no tiene & 50,0 & 35,0 & 15,0 & 100,0 \\
\hline - informal esporádico & 44,3 & 44,2 & 11,5 & 100,0 \\
\hline - informal regular & 30,8 & 44,2 & 25,0 & 100,0 \\
\hline - formal & 7,3 & 54,5 & 38,2 & 100,0 \\
\hline Total & 30,3 & 46,3 & 23,4 & 100,0 \\
\hline \multicolumn{5}{|c|}{ Pearson chi2 $(6)=27,0685$ Valor $P=0,000$} \\
\hline \multicolumn{5}{|c|}{ Calificación de los trabajadores: } \\
\hline - baja & 50,9 & 45,3 & 3,8 & 100,0 \\
\hline - media & 46,8 & 44,7 & 8,5 & 100,0 \\
\hline - alta & 9,5 & 50,0 & 40,5 & 100,0 \\
\hline Total & 31,0 & 47,3 & 21,7 & 100,0 \\
\hline \multicolumn{5}{|c|}{ Pearson chi2 $(4)=48,5641$ Valor $\mathrm{P}=0,000$} \\
\hline \multicolumn{5}{|l|}{ Metodologías ágiles: } \\
\hline - nunca & 40,6 & 50,0 & 9,4 & 100,0 \\
\hline - a veces & 35,9 & 42,3 & 21,8 & 100,0 \\
\hline - siempre valor $\mathrm{P}$ & 18,2 & 50,0 & 31,8 & 100,0 \\
\hline Total & 30,1 & 46,6 & 23,3 & 100,0 \\
\hline \multicolumn{5}{|c|}{ Pearson chi2(4) $=10,5076$ Valor $\mathrm{P}=0,033$} \\
\hline \multicolumn{5}{|l|}{ Gestión de calidad: } \\
\hline - hasta 1 esfuerzo & 45,7 & 47,8 & 6,5 & 100,0 \\
\hline - entre 2 y 3 & 41,2 & 45,6 & 13,2 & 100,0 \\
\hline - 4 ó 5 & 8,6 & 45,7 & 45,7 & 100,0 \\
\hline Total & 29,9 & 46,2 & 23,9 & 100,0 \\
\hline \multicolumn{5}{|c|}{ Pearson chi2 $(4)=40,3733$ Valor $P=0,000$} \\
\hline \multicolumn{5}{|l|}{ Certificaciones: } \\
\hline - no tiene & 47,9 & 42,2 & 9,9 & 100,0 \\
\hline - una certificación & 23,8 & 56,3 & 20,0 & 100,0 \\
\hline - dos y más & 3,1 & 33,3 & 63,6 & 100,0 \\
\hline Total & 29,3 & 46,8 & 23,9 & 100,0 \\
\hline
\end{tabular}

Pearson chi2(4) $=48,2325$ Valor $\mathrm{P}=0,000$

Recibe subsidio

\begin{tabular}{llll}
\hline & No & Sí & Total \\
\hline Gestión de calidad: & & & \\
- hasta 1 esfuerzo & 58,7 & 41,3 & 100,0 \\
- entre 2 y 3 & 29,4 & 70,6 & 100,0 \\
- 4 ó 5 & 20,0 & 79,7 & 100,0 \\
Total & 33,3 & 66,7 & 100,0 \\
\hline Pearson chi2(2)=19,0684 Valor P = 0,000 & & \\
\hline Certificaciones: & & & \\
- no tiene & 47,9 & 52,1 & 100,0 \\
- una certificación & 22,5 & 77,5 & 100,0 \\
- dos y más & 25,0 & 75,0 & 100,0 \\
Total & 32,0 & 67,2 & 100,0 \\
\hline
\end{tabular}

Pearson chi2 $(2)=12,0686$ Valor $\mathrm{P}=0,002$

Fuente: elaboración propia sobre la base de un levantamiento realizado en el marco del proyecto financiado por la Fundación Carolina.

Nota: Pearson chi2: 6, 4 ó 2 grados de libertad.

I+D: investigación y desarrollo. 
ANEXO B

\section{Estructura de la red de conexiones de las firmas}

A partir de la información obtenida en cada firma, se construyó una base de datos en que se registraron las vinculaciones orientadas a mejorar tres dimensiones de las capacidades tecno-organizacionales: i) la gestión de calidad, ii) la asistencia técnica, y iii) las actividades conjuntas de I+D. La base fue procesada utilizando el software UCINET. Esto permitió identificar en una misma red las vinculaciones que las firmas entrevistadas mantuvieron con clientes, proveedores, competidores, cámaras empresariales, organizaciones científicotecnológicas y consultores.

En el gráfico 1 se muestra la estructura de conexiones de las firmas entrevistadas. Los nodos negros representan a las empresas encuestadas y los nodos blancos a los participantes (partners) de las vinculaciones que no fueron entrevistadas.

Las firmas que se vincularon $(65 \%)$ registran un grado promedio de 2,7 conexiones por nodo.

GRÁFICO 1

\section{Red global de vinculaciones}

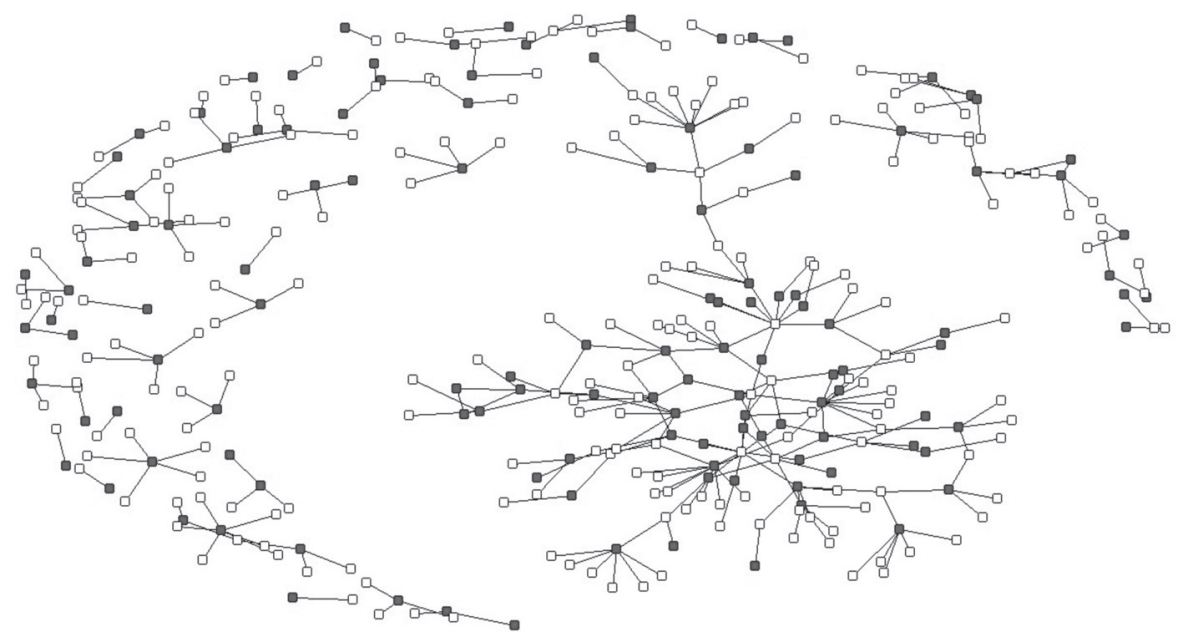

Fuente: elaboración propia sobre la base de encuesta de la Fundación Carolina. 


\section{Bibliografía}

Arora, A. y A. Gambardella (2005), From Underdogs to Tigers: The Rise and Growth of the Software Industry in Brazil, China, India, Ireland, and Israel, Nueva York, Oxford University Press.

Aspiazu, D., E. Basualdo y H. Nochteff (1990), "Los límites de las políticas industriales en un período de reestructuración regresiva: El caso de la informática en la Argentina", Desarrollo Económico, vol. 30, № 118, Buenos Aires, Instituto de Desarrollo Económico y Social (IDES).

(1986), "Estructuras y transformaciones de la industria electrónica en Argentina", Serie Documentos e Informes de Investigación, $\mathrm{N}^{\circ} 45$, Buenos Aires, Facultad Latinoamericana de Ciencias Sociales (FlaCSO).

Babini, R. (2003), La Argentina y la computadora: Crónica de una frustración, Buenos Aires, Ed. Dunken.

Barabási, A. y R. Albert (1999), "Emergence of scaling in random networks", Science, vol. 286. Nㅜ 5439, Nueva York, American Association for the Development of Science.

Borgatti, S.P., M.G. Everett y L.C. Freeman (2002), Ucinet for Windows: Software for Social Network Analysis, Harvard, Massachusetts, Analytic Technologies.

Boschma, R. y A. ter Wal (2006), "Knowledge networks and innovative performance in an industrial district: the case of a footwear district in the South of Italy", Papers in Evolutionary Economic Geography, № 601, Utrecht, Universidad de Utrecht.

Boschma, R. y A. Weterings (2005), "The effect of regional differences on the performance of software firms in the Netherlands", Journal of Economic Geography, vol. 5, $\mathrm{N}^{\circ} 5$, Oxford, Oxford University Press.

CESSI (Cámara de Empresas de Software \& Servicios Informáticos de la República Argentina) (2012), "Reporte semestral sobre el sector de software y servicios informáticos de la República Argentina. Primer semestre 2011" [en línea] http://www.cessi.org.ar/documentacion/OPPSI_ ReporteSemestralSectorSoftware_31_06_11.pdf.

Chudnovsky, D., A. López y S. Melitsko (2001), "El sector de software y servicios informáticos en la Argentina. Situación actual y perspectivas de desarrollo", Documento de Trabajo, $\mathrm{N}^{\circ} 27$, Buenos Aires, Centro de Investigaciones para la Transformación (CENIT).

Cohen, W. y D. Levinthal (1990), "Absorptive capacity: a new perspective on learning and innovation”, Administrative Science Quarterly, vol. 35, $\mathrm{N}^{\circ}$ 1, Ithaca, Universidad de Cornell.

Coombs, R. e I. Miles (2000), "Innovation, measurement and services: the new problematique", Economics of Science, Technology and Innovation, vol. 18, Springer.

Corrocher, N., L. Cusmano y A. Morrison (2009), "Modes of innovation in knowledge-intensive business services evidence from Lombardy", Journal of Evolutionary Economics, vol. 19, $\mathrm{N}^{\circ}$ 2, Springer.

Cowan, R. y N. Jonard (2004), "Network structure and the diffusion of knowledge", Journal of Economic Dynamics and Control, vol. $28, \mathrm{~N}^{\circ} 8$, junio, Amsterdam, Elsevier.

(2003), "The dynamics of collective invention", Journal of Economic Behavior \& Organization, vol. 52, $\mathrm{N}^{\circ} 4$.

Cusumano, Michael A. (2012), "Platforms versus products: observations from the literature and history", Advances in Strategic Management, vol. 29, Emerald Group Publishing.

(2010), "Cloud computing and SaaS as new computing platforms", Communications of the ACM, vol. 53, $\mathrm{N}^{\circ} 4$, abril.

Cusumano, M.A. y D.B. Yoffie (1999), "Software development on Internet time", Computer, vol. 32, $\mathrm{N}^{\circ} 10$, IEEE Computer Society.

CUTI (Cámara Uruguaya de Tecnologías de la Información) (2012) [en línea] www.cuti.org.uy.

De Bresson, C. y F. Amesse (1991), "Networks of innovators: a review and introduction to the issue", Research Policy, $\mathrm{N}^{\circ} 20$, Amsterdam, Elsevier.
Djellal, F. y F. Gallouj (2001), "Patterns of innovation organisation in service firms: portal survey results and theoretical models", Science and Public Policy, vol. 28, No 1 , Oxford University Press.

(1999), "Services and the search for relevant innovation indicators: a review of national and international surveys", Science and Public Policy, vol. 26, $\mathrm{N}^{\circ}$ 4, Oxford University Press.

Dosi, G. (2000), Innovation, Organization and Economic Dynamics: Selected Essays, Cheltenham, Edward Elgar Publishing.

Drejer, I. (2004), "Identifying innovation in surveys of services: a Schumpeterian perspective". Research Policy, vol. 33, $\mathrm{N}^{\circ} 3$, Amsterdam, Elsevier.

Freeman, C. (1991), "Networks of innovators: a synthesis of research issues", Research Policy, vol. 20, № 5, Amsterdam, Elsevier.

Gallouj, F. y M. Savona (2009), "Innovation in services: a review of the debate and a research agenda", Journal of Evolutionary Economics, vol. 19, $\mathrm{N}^{\circ} 2$, Springer.

Gallouj, F. y O. Weinstein (1997), "Innovation in services", Research Policy, vol. 26, № 4-5, Amsterdam, Elsevier.

Giuliani, E. y M. Bell (2005), "The micro-determinants of mesolevel learning and innovation: evidence from a Chilean wine cluster", Research Policy, vol. 34, $\mathrm{N}^{\circ} 1$, Amsterdam, Elsevier.

Grimaldi, R. y S. Torrisi (2001), "Codified-tacit and general-specific knowledge in the division of labour among firms: a study of the software industry", Research Policy, vol. 30, $\mathrm{N}^{\circ} 9$, Amsterdam, Elsevier.

Gulati, R. (1999), "Where do interorganizational networks come from?", American Journal of Sociology, vol. 104, $\mathrm{N}^{\circ} 5$, Chicago, University of Chicago Press.

Hargadon, A. (2003), How Breakthroughs Happen: The Surprising Truth About How Companies Innovate, Harvard Business Press.

Hughes, A. y E. Wood (2000), "Rethinking innovation comparisons between manufacturing and services: the experience of the CBR SME Surveys in the UK", Innovation Systems in the Service Economy. Measurement and Case Study Analysis, J.S. Metcalfe e I.D. Miles, Boston, Kluwer Academic Publishers.

INDEC (Instituto Nacional de Estadística y Censos) (2005), Encuesta Nacional de Innovación Tecnologica, Buenos Aires.

Klepper, S. y K.L Simons (2000), "Dominance by birthright: entry of prior radio producers and competitive and competitive ramifications in the U.S. television receiver industry", Strategic Managment Journal, vol. 21, $\mathrm{N}^{\circ}$ 10-11, John Wiley \& Sons.

Landau, R. y N. Rosenberg (1986), The Positive Sum Strategy. Harnessing Technology for Economic Growth, Washington, D.C., National Academy Press.

López, A. y D. Ramos (2007), "La industria de software en el MERCOSUR", Complementación productiva en la industria del software en los países del MERCOSUR: Impulsando la integración regional para participar en el mercado global, A. López (coord.), Documento de trabajo, $N^{\circ} 1-07$, Buenos Aires, CENIT.

Nelson, R. y S. Winter (1982), An Evolutionary Theory of Economic Change, Cambridge, Massachusetts, Harvard University Press.

oCDE (Organización de Cooperación y Desarrollo Económicos) (2002), OECD Information Technology Outlook. ICTs and the Information Economy, París.

oEdE/Mtss (Observatorio de Empleo y Dinámica Empresarial/ Ministerio de Trabajo, Empleo y Seguridad Social) (2012) [en línea] http://www.trabajo.gov.ar/left/estadisticas/oede/index.asp.

Parthasarathy, B. y Y. Aoyama (2006), "From software services to R\&D services: local entrepreneurship in the software industry in Bangalore, India”, Environment and Planning A, vol. 38, $\mathrm{N}^{\circ} 7$.

Powell, W.W., K. Koput y L. Smith-Doerr (1996), "Interorganizational collaboration and the locus of innovation: networks of learning in biotechnology", Administrative Science Quarterly, vol. 41, $\mathrm{N}^{\circ} 1$, Sage Publications. 
RAIS (Relación Anual de Informaciones Sociales) (2012), Ministerio de Trabajo y Empleo [en línea] http://www.mte.gov.br/rais/ default.asp.

Romijn, H. y M. Albaladejo (2002), "Determinants of innovation capability in small electronics and software firms in southeast England", Research Policy, vol. 31, N ${ }^{\circ}$, Amsterdam, Elsevier.

Saviotti, P.P. y J.S. Metcalfe (1984), "A theoretical approach to the construction of technological output indicators", Research Policy, vol. 13, $\mathrm{N}^{\circ} 3$, Amsterdam, Elsevier.

Savona, M. (2004), "Structural change and macro-economic performance. The structural bonus hypothesis for services", documento presentado en la XIV International Conference of RESER (Castres y Toulouse, Francia, 23-25 de septiembre).

Schilling, M.A. y C. Phelps (2004), "Interfirm collaboration networks: the impact of network structure on rates of innovation", Working Paper, Nueva York, Stern School of Business.

Segelod, E. y G. Jordan (2002), "The use and importance of external sources of knowledge in the software development process", FE rapport, $\mathrm{N}^{\circ}$ 2002-391, Göteborg, Suecia, Universidad de Göteborg.

Silverberg, G., G. Dosi y L. Orsenigo (1988), "Innovation, diversity and diffusion: a self-organisation model", The Economic Journal, vol. 98, N 393, Royal Economic Society.
Sirilli, G. y R. Evangelista (1998), "Technological innovation in services and manufacturing: results from Italian surveys", Research Policy, vol. 27, N 9, Amsterdam, Elsevier.

SOFTEX (2012), "Software e serviços de TI: A indústria brasileira em perspectiva", vol. 2 [en línea] publicacao.observatorio.softex.br/.

Verspagen, B. y G. Duysters (2004), "The small worlds of strategic technology alliances", Technovation, vol. $24, \mathrm{~N}^{\circ} 7$, Amsterdam, Elsevier.

Watts, D. (2006), Seis grados de separación: La ciencia de las redes en la era del acceso. Editorial Paidós.

(1999), "Networks, dynamics, and the small-world phenomenon", American Journal of Sociology, vol. 105.

Yoguel, G. y otros (2004), "Información y conocimiento: La difusión de las TIC en la industria manufacturera argentina", Revista de la CEPAL, $\mathrm{N}^{\circ} 82$ (LC/G.2220-P), Santiago de Chile, Comisión Económica para América Latina y el Caribe (CEPAL), abril.

Yoguel, G. y V. Robert (2010), "Capacities, processes and feedbacks: the complex dynamics of development", Seoul Journal of Economics, vol. 23, $\mathrm{N}^{\circ} 2$.

Zuckerfeld, M. y otros (2012), "Una aproximación al subsector del software y servicios informáticos (SSI) y las políticas públicas en la Argentina", presentación en el $10^{\circ}$ Simposio de la Sociedad de la Información. 